\title{
Overview on Steady-state Nuclear Methods for BWR Nuclear Core Design and Analysis
}

Ren-Tai Chiang

Energy Engineering Service, San Jose, CA 95120, USA

Corresponding author: chiang1524@gmail.com

\section{KEYWORDS}

BWR

Nuclear core design

Steady-state

\begin{abstract}
An overview on nuclear methods for boiling water reactors (BWR) core design and analysis is provided based on the ANS Standard 19.3. The steady-state BWR nuclear methods, composed of neutron cross section library generation method, lattice physics method and core physics method, are systematically reviewed and associated computer codes in common use for BWR core design and analysis are listed. Verification and validation, the two complementary aspects in determining the range of applicability of the calculation system, are discussed extensively. The biases and uncertainties for the predictions from the calculation system over its demonstrated range of applicability are also discussed.
\end{abstract}

(c) The Author(s) 2018. This article is distributed under a Creative Commons Attribution-ShareAlike 4.0 International license.

\section{INTRODUCTION}

The nuclear core design and analysis of a boiling water reactor (BWR) require knowledge of nuclear reactor physics methods and employment of associated computational software tools to calculate all nuclear regulatory complied, design-required nuclear parameters such as infinite or effective neutron multiplication factor, hot excess reactivity, cold shutdown margin, local powers, linear heat generation rate, and minimum critical power ratio at various operating state point of a specific fuel cycle.

BWR nuclear core design and analysis calculations are often performed in the following sequence (American $\mathrm{Nu}-$ clear Society 2013b):

1. Use nuclide number densities, geometrical information and neutron cross sections data to calculate the neutron spectrum for each fuel and/or core region,

2. Use the preceding spectrum to collapse multi-group neutron cross sections to few group forms for each region,

3. Use the preceding spectrum and spatial distributions to homogenize neutron cross sections over fuel rod cells or assemblies by preserving the reaction rate,

4. Use the preceding few group neutron cross sections and geometrical information about the reactor to calculate reactivity and few-group flux spatial distributions in a BWR,

5. Use the preceding information to compute reaction rates in BWR fuel and/or core components,

6. Use the preceding information to calculate changes in fuel nuclide composition with exposure.

All conditions that significantly affect the calculated quantities must be considered, which include:
1. Presence of cruciform control blades in a BWR and degradation of the effectiveness of control elements,

2. Presence and spatial distribution of Gadolinium burnable poison fuel rods,

3. Presence of adjacent, unlike fuel assemblies,

4. Composition and geometric layout of fuel in an assembly,

5. Spatial dependence of coolant/moderator density,

6. Depletion dependent conditions, including previous power history, coolant-density history, and control blade history of fuel assemblies,

7. Presence of the core shroud outside the core in a BWR,

8. Presence of sources, detectors, structural materials, and experimental devices,

9. Spatial variations in fuel and moderator temperatures,

10. Spatial and temporal variations of important nuclides, e.g., Xe-135, Sm-149, and actinides.

\section{STEADY-STATE BWR NUCLEAR METHODS}

There are three distinct aspects for BWR reactor physics analysis: neutron cross section library generation, lattice calculations, and nodal calculations. Figure 1 illustrates the sequence of such aspects and their relationship to each other (American Nuclear Society 2013b). Most of the application independent fine-group libraries for BWR applications are generated by processing data from a recent version of an evaluated data set, such as the Evaluated $\mathrm{Nu}-$ clear Data File ENDF/B-VI (Askew et al. 1966) or ENDF/BVII (Lindelow et al. 1990). These libraries typically contain 40 to 200 fine groups. The nuclide cross sections within a specific group structure are processed through the use of a "generic" BWR energy spectrum. These libraries, as shown in Figure 1, provide the cross-section data for fine-group lattice-physics calculations. 


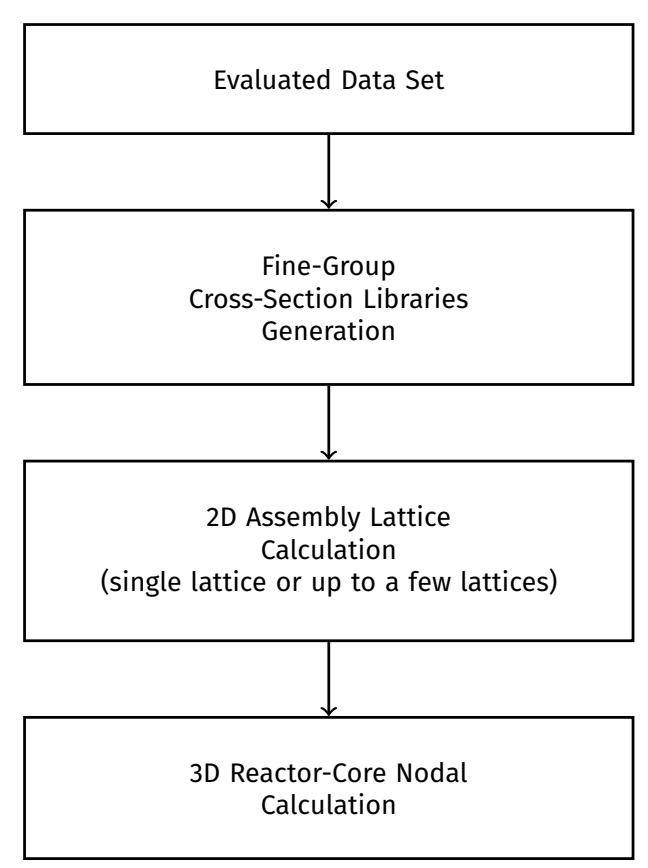

FIGURE 1. General flow of data for BWR physics calculations.

\subsection{Lattice physics method}

BWR fine- and/or few-group lattice-physics calculations typically are two-dimensional transport theory calculations that employ a fine spatial mesh (DeHart 2006). They usually are performed for a single lattice, although they may be performed for four adjacent lattice cells for specialpurpose applications. These calculations have three separate but complementary components: fine-group pin-cell calculations, intermediate-group lattice calculations, and nuclide depletion calculations. Initially, fine-group thermal and epithermal calculations are performed for the individual fuel pins or fuel-pin types and water rods or water holes, and structural components within the lattice, respectively. Fuel rods containing burnable poisons are usually modeled in greater detail and/or with more precise methods. These fine-group calculations typically employ the same energy group structure as the cross-section library. The pin cells then are homogenized, and intermediate-group cross sections for the homogenized cells are edited from the results of the pin-cell calculations. Next, intermediate-group calculations are performed for the lattice as a whole, with each homogenized pin cell retaining its own identity. Usually, both the pin-cell calculations and the intermediategroup calculations are based on transport theory. The number of energy groups in the intermediate-group calculation may range from as few as half a dozen to as many as are present in the library. Few group cross sections then are edited from the intermediate-group calculation for a homogenized representation of the lattice. Finally, nuclide depletion calculations are performed for those nuclides whose concentrations change with time, including lumped pseudo fission products. More sophisticated methods are required to calculate burnable-absorber depletion properly.

The cross sections edited from the intermediate group calculations are used as input to nodal calculations. Consequently, different sets of intermediate group calculations are performed for different combinations of thermalhydraulic variables, fuel exposure, nuclide decay during shutdown time, and control-rod state. The values of individual variations generally are chosen so that they span the range of conditions expected to be encountered during core operation.

\subsection{Core physics method}

Nodal core calculations are performed for threedimensional homogenized core analysis (Woolley 1976; Crowther et al. 1981). Individual bundles are divided into stacks of homogenized nodes, each of which typically is 10 to $30 \mathrm{~cm}$ high. Nodal calculations are based on diffusion theory. Although effective one-group nodal model that preserves the fundamental-mode multiplication factor (keff) of three-group nodal model is still in use, many now employ two or three-group advanced nodal models for this purpose. The nodal codes must account for significant thermal-hydraulic feedback into their cross sections and may include explicit nuclide depletion for actinides, major fission products, and burnable absorbers within the node. However, most current codes use "macroscopic" depletion models (macroscopic cross sections parameterized as functions of exposure, instantaneous void fraction, voidfraction history, fuel temperature, control-rod presence and control history) and treat nuclide behavior of only Xe-135 and Sm-149 explicitly.

Feedback effects between local power and moderator density (void) are prominent in BWRs (Chiang et al. 2001). Consequently, the flow distribution for the reactor core calculation, whether input or internally calculated, should correspond to a condition where the core pressure drop is the same for all parallel flow channels. It is important that all pressure drop effects and local void formation be accurately modeled. These variables strongly influence the reaction-rate distributions and reactivity of the core.

The steady-state BWR nuclear methods and associated computer codes, as shown in Table 1 (American Nuclear Society 2013b), have been utilized extensively for BWR nuclear fuel and core designs and analyses.

\section{VERIFICATION AND VALIDATION}

Verification and validation are two complementary aspects in determining the range of applicability of the calculation system. Verification assesses the fidelity of the calculation system to the theoretical models upon which it is based, while validation assesses the accuracy with which the calculation system predicts real-world behavior (American Nuclear Society 2013b). A simple example of verification is the determination of the accuracy with which a diffusion theory code produces solutions to the neutron diffusion equation, irrespective of whether diffusion theory is an appropriate representation for the behavior of interest. An example of validation is the comparison of the calculation system's predicted value for the core power distribution with the distribution that was actually measured.

The components of the calculation system shall be tested both individually and collectively. Testing of a single component is referred to as unit testing, while testing of the system as a whole is referred to as integral testing. The components of the calculation systems employed for core reactivity and power distributions for most power reactors generally fall within one of four general categories: (1) nuclear data, (2) lattice codes, (3) linkage codes, or (4) reactor core codes. Although these categories are not universal, extension to those types of reactors that have additional components should be straightforward.

Unit testing of nuclear data will not be discussed herein, as it is beyond the scope of this standard. The in- 
TABLE 1. Computer codes in common use for BWR core design and analysis.

\begin{tabular}{ll}
\hline Category & Code \\
\hline Evaluated Data Processing & NJOY (MacFarlane and Muir 1994); AMPX-2000 (Dunn and Greene 2002) \\
Unit-Cell Calculation & DRAGON (Marleau 1998) \\
Lattice Calculation & CASMO (Edenius et al. 1991); DRAGON (Marleau 1998); PHOENIX (Stammler et al. 1977); RECORD (Borresen and \\
& Skardhamar 1987); TGBLA (Chiang et al. 2001; Yamamoto et al. 1984, 1987); WIMS (Askew et al. 1966); HELIOS (Casal et al. \\
& 1991); MCNP (X-5 Monte Carlo Team 2003; Chiang et al. 2001); LANCER02 (Knott and Wehlage 2007); SCALE/TRITON \\
& (DeHart 2006); KENO (Petrie and Landers 2009) \\
Modal Diffusion Theory & MICROBURN-B2 (Lindenmeier et al. 1988); PANACEA (Woolley 1976; Crowther et al. 1981); POLCA7 (Lindelow et al. 1990); \\
& PRESTO (Borresen 1971); SIMULATE (Smith et al. 1989; Cobb et al. 1987); NESTLE (Turinsky et al. 2003); AETNA02 (Palmtag \\
& et al. 2008); PARCS (Downar et al. 2009) \\
Transport Theory & TORT (Rhoades and Childs 1987); DORT (Rhoades and Childs 1988) \\
Monte Carlo & MCNP (DeHart 2006); KENO (Petrie and Landers 2009) \\
\hline
\end{tabular}

terested reader is referred instead to ANSI/ANS-19.1-2002; R2011 (American Nuclear Society 2013a).

\subsection{Verification}

Verification requires comparisons with closed-form analytic solutions or reference results from a previously verified code. Verification typically is performed by the code developer, with the results included within the documentation distributed with the code.

Analytic and/or simplified artificial benchmarks are the primary tool employed for unit testing within the context of verification. In general, verification is a binary process: the code either replicates the reference result to an acceptably fine level of precision or it does not. If it does not, the coding shall be examined until the reasons for the discrepancies are identified and understood. If these reasons are found to be errors in the code, the code shall be revised, and the verification process shall be repeated.

Unit testing of lattice codes poses a number of challenges, foremost of which is the construction of an artificial nuclear data library in the format required by the code (MacFarlane and Muir 1994). In such cases, it is acceptable to extract the "solver" portion of the code and use it with a front-end driver that produces the cross sections, nuclides, and geometry information required by the solver.

In most such cases, the benchmark documentation includes results from a number of lattice codes, and the results from the lattice code of interest can be compared both to the reference results and to the results obtained by other codes based on the same methodology. In addition, with the advent of computers with large amounts of memory, lattice codes sometimes may be applied to benchmarks that formerly were employed only for reactor core codes. The most likely of these benchmarks are those that have a twodimensional representation of a symmetric fraction of a reactor core (Petrie and Landers 2009).

Although unit testing of lattice codes usually is thought of in terms of reactivity and power distributions, it also shall include testing for nuclide production and depletion. No matter how accurately a lattice code calculates the flux distribution, its predictions for reactivity and power distributions will be degraded with burnup if it does not also calculate nuclide production and depletion correctly.

The purpose of the linkage code is to transform the output from the lattice-code calculations (homogenized cross sections, etc.) into input for the reactor core code. The accuracy of this transformation can be assessed by comparing the results from the lattice-code calculations with the input as reconstructed by the reactor-core code. Given cri- teria for acceptable differences, such comparisons not only satisfy the requirements for unit testing of the linkage code but also provide information for guidelines about the length of burnup steps for the lattice-code calculations.

A number of benchmarks exist for integral testing of reactor-core codes, complete with macroscopic cross sections. Integral testing for verification purposes is a difficult task, because there are few appropriate benchmarks for nuclear systems that encompass the full range of the type of analyses that are performed by the calculation systems of interest in this standard.

\subsection{Validation}

Validation requires comparisons with real-world measurements, such as those from critical experiments or operating reactors. Typically, the code developer will perform much of the validation, with the results included within the documentation distributed with the code. Further validation against results from the operating reactor of interest or a very similar reactor should also be performed before the calculation system is employed to model that reactor on a routine basis.

Unit testing for validation purposes may be performed in either of two ways. The most direct is to compare code predictions directly to measured results. However, measured results typically are integral rather than unit parameters. A second approach is to compare predictions from the code of interest with results from a code of comparable or better accuracy that already has undergone verification and validation.

Lattice codes perform calculations in two dimensions rather than three dimensions. Consequently, comparisons with measured data are usually made for zero-power rather than at-power conditions so that three-dimensional effects may be treated in some accurate but relatively simple manner (e.g., assigning an input value for axial buckling) (Borresen 1971; Turinsky et al. 2003). Critical experiments are good candidates for unit testing of lattice codes so long as geometric distortions are minimal.

Unit testing for lattice codes may also be performed by comparing their predictions with results from another code of comparable or better accuracy. Monte Carlo codes often serve this function. It must be emphasized that the nuclear data libraries of the two codes must be sufficiently similar that the differences observed are due to the two codes themselves and not to their libraries (Petrie and Landers 2009). In practice, this restriction means that the two nuclear data libraries are derived from the same version of the same evaluated data set. 
The purpose of the linkage codes is to transform output from lattice-code calculations into input for reactor-core codes. Consequently, there is no need for unit testing for validation of linkage codes.

Reactor core code relies on input from lattice-code calculations and cannot perform calculations for real-world applications without that input. Consequently, unit testing of a reactor core code for validation cannot be performed against measured data unless that code represents each fuel pin, absorber pin, water hole, etc. explicitly. In that case, unit testing and integral testing become identical.

Integral testing for validation purposes requires comparisons with measurements from operating reactors. In these comparisons, due consideration must be given to uncertainties or tolerances in enrichment, composition, densities, as-built dimensions, and other engineering state variables. In addition, results from critical experiments or from more rigorous codes may be used to supplement these comparisons when appropriate.

In most cases, prior to the release of the code, the code developer performs calculations for a number of reactors over several cycles and compares the predicted and measured reactivity worth and power distributions. Such comparisons establish general guidelines for the accuracy that may be expected when the code is applied to similar calculations for reactors of the same general type.

If the calculation system is to be used for licensing purposes or for in-core fuel management, similar comparisons should be made with measurements for the specific reactor of interest. If the reactor has not yet begun operation, such comparisons should be made with measurements from other reactors that are very similar to the one of interest. Furthermore, it is imperative that the model employed during integral testing be exactly the same as that which will be used for routine applications.

The advantage of using results from critical experiments is that the uncertainties associated with such measurements usually are smaller and more readily quantifiable than those from operating reactors, even when the reactors are at zero-power conditions (Dunn and Greene 2002). On the other hand, critical experiments typically are performed at room temperature and have little if any thermalhydraulic feedback (Marleau 1998). In addition, critical experiments are usually limited to zero-burnup fuel, and they often feature different geometries from those in power reactors, for both the fuel design and the core arrangement.

In addition, comparisons and benchmarking may be made with results from other codes, of comparable or greater accuracy, that have already undergone verification and validation. At the present time, most reactor-core codes are based on some variation of neutron diffusion theory. Consequently, codes based on transport theory (e.g., Monte Carlo or nodal transport codes) may satisfy this objective. However, two restrictions must apply. First, there shall be no overlap in the components of the two systems, except for nuclear data libraries. Second, the nuclear data libraries employed by the two systems must be sufficiently similar that the differences observed are due to the codes themselves and not to their libraries. In practice, this restriction means that the two nuclear data libraries are derived from the same version of the same evaluated data set and requires that the equivalence is proven.

\section{BIASES AND UNCERTAINTIES}

The results from the validation process, particularly integral testing, can be used to establish biases and uncertain- ties for the predictions from the calculation system over its demonstrated range of applicability (American Nuclear Society 2013a). In addition, confidence levels shall be specified for the established uncertainties.

If the calculation system is applied beyond its demonstrated range of applicability, the assigned uncertainties shall be increased by an amount that reflects the extent of the extrapolation and the possibility that other factors or phenomena can influence the results obtained. However, extending the use of the code system to a new range of applicability will require additional validation of the system in the new area of applicability for licensing applications.

\section{CONCLUSIONS}

An overview on nuclear methods for Boiling Water Reactors (BWR) core design and analysis has been provided based on the ANS Standard 19.3. The steady-state BWR nuclear methods, composed of neutron cross section library generation method, lattice physics method and core physics method, have been systematically reviewed and associated computer codes in common use for BWR core design and analysis have been listed. Verification and validation are the two complementary aspects in determining the range of applicability of the calculation system and have been discussed extensively. The biases and uncertainties for the predictions from the calculation system over its demonstrated range of applicability have also been discussed.

\section{REFERENCES}

American Nuclear Society. 2013a. Ans 19.1:2002(R2011): Nuclear data sets for reactor design calculations. American Nuclear Society.

American Nuclear Society. 2013b. ANS 19.3:2011(R2017): Steady-State Neutronics Methods for Power Reactor Analysis. American Nuclear Society.

Askew JR, Fayers FJ, Kemshell PB. 1966. General description of the lattice code WIMS. J Brit Nucl Energy Soc. 5:564585.

Borresen S. 1971. A simplified, coarse mesh, three dimensional diffusion scheme for calculating the gross power distribution in a boiling water reactor. Nucl Sci Eng. 44:37-44.

Borresen S, Skardhamar T. 1987. Recent developments in the lattice physics code RECORD. volume 3. Paris.

Casal J, Stammler R, Villarino E, Ferri AA. 1991. HELIOS: geometric capabilities of a new fuel assembly program. In: Proc Int Topl Mtg Advances in Mathematics, Computations, and Reactor Physics. volume 5. Pittsburgh. p. 13.

Chiang RT, Sitaraman S, Jenkins A, Asano K, Koyabu K. 2001. Development, validation and application of innovativeMNCP-based BWR fluence computational package MF3D. In: $9^{\text {th }}$ Int Conf on Nuclear Engineering. Nice. p. 8-12.

Cobb WR, Borland RS, Darnell BL, Versteegen PL, Ver Planck DM. 1987. ARMP-02 documentation: Part 2, Chapter 8, SIMULATE-E (Mod. 3) computer code manual. Technical Report EPRI-NP-4574-CCM-PT.2-CH.8. Science Applications.

Crowther R, Congdon S, Crawford B, Kang C, Leonard A, Martin C, McDaniel C, Schwager L, Walter RA. 1981. Three dimensional simulation of large power reactors. In: Proceedings of International Topical Meeting on Advances in Mathematical Methods for the Solution of $\mathrm{Nu}^{-}$ clear Engineering Problems. Munich. 
DeHart MD. 2006. TRITON: a two-dimensional depletion sequence for characterization of spent nuclear fuel. Technical Report ORNL/TM-2005/39 Version 6, Vols. I-III. Oak Ridge National Laboratory. Oak Ridge.

Downar T, Yu X, Seker V. 2009. PARCS v3.0 Theory Manual. Technical report. University of Michigan. Ann Arbor.

Dunn M, Greene M. 2002. Cross-section processing system for generating nuclear data for criticality safety applications. Trans Am Nucl Soc. 86:118-119.

Edenius M, Forssen B, Gragg aC. 1991. The physics model of CASMO 4. In: Proc Int Topl Mtg Advances in Mathematics, Computations, and Reactor Physics. volume 2. Pittsburgh.

Knott D, Wehlage E. 2007. Description of the LANCR02 lattice physics code for single-assembly and multi-bundle analysis. Nucl Sci Eng. 155(3):331-354. doi:10.13182/nse 155-331.

Lindelow U, Jonsson C, Lindahl aSO. 1990. The ABB atom PWR core design code package. In: Reactor Core Design and Performance Evaluation Methods, ASEA Atom Report RCA. volume 77. Nuremberg. p. 15.

Lindenmeier C, Timmons D, Correll GR. 1988. Advanced methodology for LWR core design. In: Proc. of the 1988 International Reactor Physics Conference. volume 1. Jackson Hole.

MacFarlane RE, Muir DW. 1994. The NJOY nuclear data processing system Version 91. Technical Report LA-12740M. Los Alamos National Laboratory. Los Alamos.

Marleau G. 1998. Fine mesh 3-D collision probability calculations using the lattice code DRAGON. In: International Conference on the Physics of Nuclear Science and Technology. Long Island. p. 523-530.

Palmtag S, Lamas J, Finch J, Godfrey A, Moore BR. 2008. The advanced BWR core simulator AETNA02. http://inis.i aea.org/Search/search.aspx?orig_q=RN:41116214.

Petrie LM, Landers NF. 2009. KENO V.a: an improved Monte Carlo criticality program with supergrouping.
Ver. 6. Technical Report NUREG/CR-0200-VOL.2. Oak Ridge National Laboratory. Oak Ridge.

Rhoades WA, Childs RL. 1987. The TORT three-dimensional discrete ordinates neutron/photon transport code. Technical Report ORNL-6268. Oak Ridge National Laboratory. Oak Ridge.

Rhoades WA, Childs RL. 1988. The DORT two-dimensional discrete ordinates transport code. Nucl Sci Eng. 99(1):88-89. doi:10.13182/NSE88-A23547.

Smith KS, Rempe KR, VerPlanck DM. 1989. SIMULATE-3 Methodology: Advanced three-dimensional two group reactor analysis code. Technical report. Studsvik of America, Inc. Newton.

Stammler R, Olsson S, Bernander Ö. 1977. The PHOENIX computer program for fuel assembly and pin cell calculations. Technical Report ASEA ATOM PM RCA 77-012.

Turinsky P, Al-Chalabi R, Engrand P, Sarsour H, Faure F, Guo W. 2003. NESTLE Version 5.2.1: Few-group neutron diffusion equation solver utilizing the nodal expansion method for eigenvalue, adjoint, fixed-source steady-state and transient problems. Technical report. North Carolina State University. Raleigh. doi:10.2172/ 10191160.

Woolley JA. 1976. Three-dimensional BWR core simulator. Boiling Water Reactor Systems Dept., General Electric Company.

X-5 Monte Carlo Team. 2003. MCNP-a general Monte Carlo $\mathrm{N}$-particle transport code, version 5. https://mcnp.l anl.gov/monp5.shtml.

Yamamoto M, Ando Y, Unamo T, Mizuta H, Seino H. 1987. Recent developments in TGBLA lattice physics code. In: Proc Int Topl Mtg Advances in Reactor Physics, Mathematics, and Computations. volume 3. Paris.

Yamamoto M, Mizuta H, Makino K, Chiang RT. 1984. Development, and validation of TGBLA BWR lattice physics methods,. In: Proc Topl Mtg on Reactor Physics and Shielding. volume 1. Chicago. 